\title{
Socio-anthropologie d'une expertise : cas d'une évaluation externe d'un projet au Niger
}

Sanda Maman Sani

\section{OpenEdition}

\section{Journals}

Édition électronique

URL : http://journals.openedition.org/apad/1942

DOI : 10.4000/apad. 1942

ISSN : 1950-6929

Éditeur

LIT Verlag

Édition imprimée

Date de publication : 1 décembre 1994

Référence électronique

Sanda Maman Sani, «Socio-anthropologie d'une expertise : cas d'une évaluation externe d'un projet au Niger », Bulletin de l'APAD [En ligne], 8 | 1994, mis en ligne le 23 novembre 2007, consulté le 08 septembre 2020. URL : http://journals.openedition.org/apad/1942 ; DOI : https://doi.org/10.4000/ apad. 1942

Ce document a été généré automatiquement le 8 septembre 2020

Bulletin de I'APAD 


\title{
Socio-anthropologie d'une expertise : cas d'une évaluation externe d'un projet au Niger
}

\author{
Sanda Maman Sani
}

Introduction

1 Durant mon séjour sur le terrain de recherche, une occasion s'était présentée de suivre, en tant qu'anthropologue-observateur muet, les travaux d'évaluation externe du projet sur lequel je travaillais. Il m'était donc donné de mesurer la nature et les raisons possibles de l'écart entre ce que les experts évaluateurs d'un projet peuvent voir et entendre durant leur court séjour et ce qui se passe et est dit par les acteurs.

2 La présence de J.-P. Olivier de Sardan à Niamey a facilité les démarches en vue d'avoir l'accord des différentes parties: Mission Française de Coopération, Caisse Française de Coopération, P.A.G.T. et les évaluateurs. Les différentes parties ont spontanément accepté cette proposition.

3 L'objectif principal qui m'a guidé dans ce travail était de "démontrer la factibilité et l'intérêt d'une évaluation qualitative donnant la parole aux ruraux et élaborer un outil méthodologique permettant d'améliorer la préparation, la conduite et l'impact des opérations de développement" (T. Bierschenk).

4 Il s'agissait donc de conduire une évaluation pilote utilisant des outils appartenant à l'anthropologie du développement (enquête qualitative longue sur le terrain, implication de chercheurs expérimentés travaillant directement en langue locale et prise en compte de tous les acteurs concernés).

5 Cette approche basée sur l'insertion progressive d'un observateur extérieur participant de la même culture et de la même langue devrait permettre de réduire le biais introduit automatiquement lors des évaluations classiques réalisées par des opérateurs assimilés au projet, à l'administration ou aux bailleurs de fonds.

6 En effet la multiplication des études de projets et l'utilisation de techniques sophistiquées ne parviennent pas à expliquer ex-post de façon pleinement satisfaisante 
et surtout à prévoir ex-ante le succès ou l'échec d'une opération de développement Cela peut s'expliquer en partie par une insuffisance majeure dans le mode de conduite des évaluations (ex-ante comme ex-post) en milieu rural.

7 Ex-ante :

- Les évaluations sont encore souvent dominées par des considérations techniques ou prisonnières des exigences formelles des bailleurs de fonds.

- La soi-disant prise en compte des "besoins réels exprimés par les populations" se réduit en fait à la superposition de deux images subjectives : celle que les ruraux se font de ce que les intervenants sont susceptibles d'offrir et celle que les intervenants eux-mêmes se font des "populations cibles".

8 La seule référence à un financeur potentiel introduit un biais important qui modifie les stratégies de toutes les parties concernées.

Ex-post, les évaluations pratiquées entrent dans la typologie suivante :

- Auto-évaluation bienveillante par l'opérateur lui-même, par une administration dont il est un démembrement ou par le bailleur de fonds.

- Évaluation externe menée par un opérateur professionnel indépendant mais reposant principalement sur les rapports et informations officiels du projet et des visites de terrain effectuées au pas de course en compagnie de l'encadrement.

-Évaluation plus fine associant une comparaison quantitative "prévisions / réalisations" (bâtiments construits, nombre de paysans formés, kilomètres de banquettes anti-érosives) avec une étude d'impact socio-économique plus qualitative (évolution des productions, des revenus, de l'emploi, de l'équilibre alimentaire).

10 Dans tous ces cas l'opinion qu'ont les acteurs ruraux (producteurs mais aussi opérateurs économiques ou chefferie traditionnelle) sur des opérations qui les concernent directement ainsi que sur les conditions ou les causes de leur réussite ou de leur échec est inconnue, marginalisée ou profondément biaisée lors de sa prise en compte. (J .P. olivier de Sardan, D. Mignot (1993)).

C'est sur la base de ces constats que j'ai entrepris un travail de recherche sur un terrain circonscrit (une zone agro-pastorale, canton de Torodi) qui a connu diverses opérations de développement (dont le projet forestier). Certaines de ces opérations sont encore récentes et présentes sur le terrain .C'est le cas du projet appui à la gestion de terroirs (PAGT), projet sur lequel je me suis basé pour expérimenter ma démarche. Cela a été possible grâce au soutien de la Caisse Française de Développement (CFD) et le Fond d'Aide et de Coopération (FAC).

12 C'est donc fort de tout ceci que je me suis installé sur le terrain de l'action pour une durée de 4 mois, en vue d'étudier l'image que se font les populations-cibles du dit projet et de ces agents.

13 Ainsi, pour comprendre la logique qui a guidé cette mission tout au long de son déroulement, j'ai jugé utile de présenter sa composition et son chrono gramme avant l'analyse des résultats auxquels elle a abouti.

Cette mission d'évaluation était composée :

- d'un expert international, agro-économiste spécialisé dans les systèmes de production vivriers sahéliens, expert français recruté par la S.A.T.E.C.,

- d'un expert international, spécialiste de l'animation, de auto promotion rurale et de l'appui à l'émergence d'organisations rurales et professionnelles, expert français recruté par la S.A.T.E.C., 
- et d'un expert national, aménagiste (techniques d'aménagement adaptés à une démarche participative en milieu sahélien), Nigérien recruté par un bureau d'étude de la place.

Évaluation externe de projet : contexte et contraintesEmploi du temps

- Premier jour : rencontre avec les bailleurs de fonds, le staff technique du projet et

l'administration nigérienne.

- Deuxième jour : consultation de l'ensemble des documents relatifs au projet

- Troisième jour : organisation du travail

Avant d'établir leur programme, la mission avait émis un souhait de prolongation de la durée de l'évaluation. Le Conseiller technique représentant du F.A.C. et le représentant de la Caisse Française de Coopération avaient pris acte et promis d'en parler aux responsables des institutions respectives.

C.F.D. avait répondu favorablement, tandis qu'au niveau de la mission française de coopération, il avait été dit que cette proposition, si elle était retenue, ferait l'objet d'un nouveau dossier de commande d'étude auprès de la S.A.T.E.C. Suggestion que les membres de la mission d'évaluation avaient rejetée pour des raisons liées à la lourdeur administrative française, car selon eux, si l'on décidait d'introduire un nouveau dossier à cette date là, il faudrait attendre près de six mois pour obtenir un déblocage des fonds. C'est ainsi qu'ils ont décidé de respecter la limite de temps initialement prévue. Le programme avait été établi comme suit : - trois jours de terrain (site du projet à soixante kilomètres environ de Niamey). A son arrivée, elle rencontra à 9 heures les cadres et animateurs du P.A.G.T. en vue de la préparation avec ces derniers de la visite de terrain.

Suite à cette rencontre, un programme détaillé de visite des villages avait été élaboré en vue de permettre aux animateurs de descendre passer l'information aux villageois.

21 Et c'est 10 heures 30 que la mission s'est scindée en trois groupes pour effectuer les visites. Ainsi les deux experts français se sont partagé les 11 villages, l'un en visitant 6 , l'autre les 5 restants; chacun devait faire un village le matin et un autre le soir. L'expert nigérien, quant à lui, devait par contre visiter la totalité des villages, car il devait inspecter toutes les installations en hydraulique villageoise et en CES/DRS.

Ainsi, il avait été prévu de visiter l'ensemble des villages (11) de la zone pilote en 3 jours, car disaient-ils, comme l'échantillon est petit, la visite de toutes les réalisations était nécessaire.

Méthode d'approche de terrain

Partout où ils sont passés, les missionnaires ont posé les questions suivantes :

- depuis quand le projet s'est-il installé ?

- comment les villageois jugent-ils le P.A.G.T.?

- qu'attendent-ils du P.A.G.T.?

- ont-ils connu d'autres opérations de développement avant le P.A.G.T. ?

- comment imaginent-ils l'évolution future du projets ?

- comment comptent-ils gérer les réalisations faites avec l'appui du P.A.G.T. après la fin de ce dernier?

D'une manière générale, l'assistance était composée du chef de village ou de son représentant, des membres du Bureau de la coopérative (dans le cas de l'ancienne 
zone), du gérant de la RC., du secouriste, de la matrone, des instructeurs en alphabétisation et de quelques villageois (en général 4 à 5 personnes). aussi permis à ces derniers de développer un discours qui allait dans le sens d'une captation future des ressources financières du P.A.G.T., car jusque là, ils n'avaient pas profité de ce dernier comme ils le faisaient avec les projets classiques. Cette stratégie n'a pas marché avec les évaluateurs qui ont voulu rester fidèles à l'esprit de la démarche gestion des terroirs qui consiste à traiter directement avec les villageois sans passer par des intermédiaires. Néanmoins, il semble que des possibilités de contrats existent entre le projet et les services techniques. séjour de la mission. Cela s'explique à notre avis par leur statut de fonctionnaires détachés. Aussi il nous semble que leur groupe ait été le seul à garder à l'esprit l'échéance de la fin prochaine du projet Pour eux, comme pour les animateurs, l'image qu'ils avaient des évaluateurs était la même : "les évaluateurs et les bailleurs de fonds sont les mêmes personnes, car même nos patrons ont peur d'eux" m'a déclaré un cadre du P.A.G.T.

relations que les cadres de la cellule centrale ont entretenues avec les évaluateurs ont été des-relations intéressées. Cela se comprend aisément car ces cadres étaient les "grands penseurs" chargés d'apporter un appui méthodologique à l'équipe de terrain, donc eux aussi attendaient des évaluateurs une certaine reconnaissance du travail accompli. C'est sûrement pour cette raison qu'ils avaient tenu à donner de plus amples explications sur les difficultés auxquelles ils avaient été confronté tout le long de la durée de vie du P.A.G.T..

33 Face aux évaluateurs, les bailleurs de fonds ont beaucoup insisté sur le respect des termes de référence et une obligation de résultats. Leur réticence quant à la 
prolongation automatique de la durée de la mission a fait dire au chef de celle-ci, que les bailleurs de fonds avaient déjà pris leur décision et qu'ils attendaient d'eux des éléments la confortant.

Les villageois n'ont pas tout à fait échappé aux jeux classiques des animateurs qui pour sauver leur projet, leur boulot, ont mobilisé certains leaders ayant des intérêts dans le projet afin de présenter aux évaluateurs le show habituel que l'on offre aux visiteurs (défilé des leaders villageois impliqués dans le projet).

Certains animateurs ont su jouer le jeu avec une très grande habileté, de telle sorte que l'observateur muet que nous étions n'a compris que beaucoup plus tard la manœuvre.

De la réaction des villageois face aux évaluateurs

Dans pratiquement tous les villages de l'ancienne zone, les réponses aux questions posées par les évaluateurs étaient les mêmes. Elles se résument comme l'a constaté le Chef de la mission d'évaluation aux préoccupations à court terme, des problèmes sur lesquels les villageois butent tous les jours et qu'ils veulent résoudre.

Il faut dire aussi que malgré la minutieuse stratégie de préparation des villageois qui avait été mise en place par les animateurs de certains villages, les villageois n'ont pas toujours respecté le pacte (qui consiste à restituer un discours susceptible d'influencer les évaluateurs en faveur de la poursuite du projet). Cela a d'ailleurs provoqué une certaine polémique entre les villageois de Niakatiré (un village du projet) et leurs animateurs, car suite à la question posée par un membre de la mission sur les problèmes que les villageois rencontrent avec l'encadrement du P.A.G.T., un jeune villageois aurait voulu répondre objectivement, mais l'animateur a protesté (toujours en langue locale), lui disant qu'il y a " des choses qu'on ne doit pas dire aux Blancs. Même si elles sont vraies, elles doivent être laissées à l'appréciation des nationaux. C'est une cuisine interne, attendons le départ des Blancs pour parler de ça ". Ce discours a naturellement échappé à l'évaluateur car il n'a jamais été traduit.

Synthèse et restitution Exposé des résultats

La restitution orale des premiers résultats (en l'absence de toute personne de Torodi, que ce soit responsables paysans, animateurs ou cadres du projet), juste après les visites des sites et les rencontres avec les différents partenaires a constitué l'étape la plus importante du travail réalisée par la mission d'évaluation.

La réunion a regroupé :

- les représentants des bailleurs de fonds ;

- le staff technique du projet (uniquement les responsables de la cellule centrale);

- les représentants de l'administration nigérienne.

Après une brève description du projet et de ses activités, la mission par la voix de son chef a tiré les conclusions suivantes ${ }^{1}$ :

Le projet P.A.G.T. a souffert :

- de la divergence et de la diversité des tâches assignées ;

- de retards (largement dus aux bailleurs de fonds) dans sa mise en œuvre ;

- d'un cadre institutionnel inadéquat ;

- d'une carence des procédures de gestion administrative et financière ;

- d'une absence de programme de formation ;

- d'une définition approximative des contraintes opérationnelles. 
Sur la base de telles conclusions, le projet n'a pas d'avenir. Cependant la mission souligne qu'il y a des éléments positifs qui permettent d'envisager une poursuite de façon différente.

Selon la mission, les conditions d'une telle poursuite sont :

a) La mise en place d'un projet pour une période de 10 ans avec deux phases quinquennales évaluées à mi-parcours.

b) Travailler à l'échelle de tout le canton (95 villages) avec une stratégie d'intégration progressive et sans complaisance des villages les plus susceptibles d'adhérer à la démarche.

c) Un personnel évolué et formé avec un encadrement lui-même restructuré et formé.

d) Des diagnostics villageois entièrement repensés pour permettre une meilleure efficience des personnels et un suivi et une évaluation (y compris auto-évaluation) de qualité.

e) Une direction de projet maîtrisant la démarche et basée à Torodi avec une autonomie administrative et financière qui laisserait à cette direction le choix de ses moyens et notamment le recrutement du personnel.

Réaction des différentes parties : conflit des logiques

À la fin de l'exposé, les réactions des différents partenaires ne se sont pas fait attendre.

C'est ainsi que le Directeur du P.A.G.T. a pris la parole pour rappeler à l'assistance les conditions combien difficiles dans lesquelles l'ensemble de l'équipe du projet a eu à travailler. Après quoi, il a fait état de son indignation en apprenant que le projet n'a pas d'avenir.

L'expert nigérien a pris la parole à son tour pour réconforter la direction du P.A.G.T. en affirmant qu'en l'état actuel des choses, les paysans adhéraient à la démarche et s'inquiétaient de la menace d'arrêt des activités du projet. Il existe un très grand risque, ajouta-t-il, en cas de rupture des activités, surtout dans le cadre des travaux C.E.S/D.R.S. où des perspectives meilleures existent. Le projet était un espace d'expérimentation et de recherche dans la phase actuelle.

51 Un autre représentant de la partie nigérienne, en l'occurrence le représentant du MHE, a pris la parole pour expliquer que la mouvance en matière de GT permettait d'espérer des perspectives meilleures malgré tous les problèmes rencontrés par le P.A.G.T.

De ces trois interventions s'est dégagée une position en faveur d'une poursuite de l'opération telle qu'elle.

3 C'est alors que le représentant de la Caisse Française de Développement a fait remarquer qu'il est vrai que le P.A.G.T. a souffert de ses objectifs, notamment dans les deux tâches de la cellule centrale (appui et capitalisation), mais si l'on devait prolonger l'opération, il faudrait des objectifs beaucoup plus clairs avec un autre projet.

54 Le chef de mission de coopération française a eu à attirer l'attention des évaluateurs sur les contraintes qui pouvaient surgir dans les procédures de financement d'un projet autonome, écartant par là-même la question de l'autonomie d'une future opération.

Le Conseiller technique du P.A.G.T. a quant à lui pris la parole pour poser les questions suivantes : comment le désengagement sera-t-il géré (arrêt du projet) ?, comment insuffler une dynamique supplémentaire (poursuite du projet) ?, et comment apporter les rectifications nécessaires (modification du projet actuel)? 

ici par la demande de participation d'une cellule du ministère aux travaux de la mision d'évaluation; cela dans le seul but, à notre avis, d'avoir une main-mise sur celle-ci, de peur de perdre le contrôle de certains niveaux de décision d'orientation de l'aide.

64 * Ainsi dès au départ l'administration centrale a voulu utiliser une stratégie de récupération ou de détournement d'une mission qui se voulait indépendante et externe.

$65 *$ Les bailleurs-de fonds semblent eux aussi utiliser une stratégie de légitimation de leur décision de se désengager du financement de cette forme d'aide. Ici cette stratégie a été de préparer des termes de référence avec des objectifs précis et des a priori auxquels les experts étaient "tenus" de répondre.

66 Conséquence : bailleurs de fonds et experts ont fait front commun pour défendre l'esprit d'indépendance de la mission, donc son contrôle avait complètement échappé à l'administration nigérienne.

2) Problème lié à la collaboration entre experts du Nord et ceux du Sud.

Dans ce cas précis l'expert nigérien nous a semblé mal à l'aise tout au long de la mission, cela pour trois raisons principales :

- il se disait isolé dans les rapports de l'équipe avec les bailleurs de fonds ; en effet, l'expert nigérien se disait quelque peu écarté dès au départ des grandes décisions. Il n'avait, semblait-il, pas été associé à certaines rencontres que ses deux collègues avaient eu avec les bailleurs de fonds pour décider de la méthode de travail et des objectifs de l'évaluation.

- il donnait l'impression de porter deux casquettes, celle d'expert indépendant et celle de patriote devant défendre son pays dans la stratégie de captation de l'aide ; c'est du moins les conclusions auxquelles nous avons abouti après analyse de son comportement sur le terrain et de ses positions lors de la restitution orale des premiers résultats.

Bulletin de l'APAD, 8 | 1994 
• enfin, il se sentait gêné de "rendre compte" à ses collègues français, car il devait envoyer son rapport à ces derniers en France pour qu'ils l'intègrent dans le rapport de l'équipe. Ce qu'il n'a semble-t-il pas fait, entraînant ainsi la production de deux rapports différents.

Conséquence : il se pose là un véritable problème de collaboration qui laisse apparaître une stratégie pour les experts nationaux de vouloir contrôler le marché local là où les experts du Nord cherchent à en garder le contrôle.

3) Problème lié au biais induits par la traduction faite par les animateurs du projet.

Les animateurs représentent les acteurs les plus proches des bénéficiaires dans l'encadrement des projets. Ils sont en partie responsables de certaine réussite du projet. Mais aussi (ce qui à notre avis est le plus important) ils ne sont jamais neutres. Ils développent des stratégies de manipulation des populations dans la restitution des informations relatives aux résultats de leur travail.

Dans ce cas précis les animateurs n'ont pas raté l'occasion, car dans presque tous les villages où les experts français sont passés seuls (sans l'expert nigérien) l'animateur qui leur sert d'interprète a soigneusement transformé les "dires" des paysans en produisant un discours très proche de ce qui pouvait faire croire à ses interlocuteurs que les choses se présentent très bien. Mieux, les animateurs ont réussi à utiliser la même stratégie face à l'expert nigérien (supposé parler la langue locale) en se servant des paysans de contact (ou paysans de relais ou encore animateurs ruraux) qui se sont spécialisés dans la restitution du discours officiel (en langue locale) du projet C'étaient eux qui prenaient la parole pour répondre à l'expert nigérien.

Remarques : être expert local et maîtriser la langue des bénéficiaires n'est pas en soi suffisant pour contrôler les stratégies de certains acteurs sur le terrain.

4) Problème lié à l'utilisation par les agents de développement des méthodes de travail enseignées par les institutions de formation dans le monde rural (E.N.D.A, C. S. A.O, C.E. P.E .A.C etc.)

5 Le rôle joué par ces institutions dans le cas du PAGT a été d'aider les agents de développement à mettre une véritable démarche de gestion de terroirs en leur fournissant les principaux outils de travail avec les ruraux. Ici les agents ont réussi à se réapproprier une technique assez facile de restitution du contenu d'un programme des différentes phases de sa mise en œuvre. Cette technique a permis à toute l'équipe du projet de mettre en place une stratégie de mise en scène à travers des panneaux entreposés dans une grande salle au siège du projet sur lesquels on présente aux visiteurs la philosophie et les différentes phases du projet. (en français). La même technique est utilisée au niveau des villages P.V.D avec des panneaux en langue locale.

Remarques : malgré tout les évaluateurs n'ont pas été dupes car il semble que ce n'est pas la première fois qu'ils rencontrent des situations où les méthodes d'approche du monde rural enseignées par les institutions de formation sont détournées pour des mises en scène par les agents de développement. Ils n'ont d'ailleurs pas manqué de souligner au chef de zone pilote chargé de leur présenter les panneaux.

5) Problème lié au temps

Trois jours seulement avec des entretiens d'une à deux heures par village et une heure de site. Caractère expéditif de l'évaluation externe dû à la brièveté du séjour des experts. 
79 6) Ainsi j'ai assisté tout le long de son déroulement sur le terrain à un refus catégorique de certains animateurs à traduire certaines réponses des paysans. Tout le discours paysan lié au projet et à sa démarche à complètement échappé aux membres de cette mission. L'exemple le plus frappant est celui d'un villageois qui a été applaudi par les membres de son groupement lorsqu'il disait ceci:"le projet IDA (projet forestier ,précédant le PAGT) était l'étranger qui avait offert une canne à un vieillard fatigué pour lui permettre de se relever, alors que le PAGT est considéré comme un étranger qui ne tend pas la canne au même vieillard mais la jette pour lui demander de faire un effort pour la reprendre ". Nous sommes donc là en présence d'un discours assez important, opposant un projet d'assistance et un projet d'appui aux initiatives locales, ce qui traduit à n'en point douter un retard dans l'appropriation par les populations cibles de la démarche même du projet. A notre avis seule une présence d'une durée assez raisonnable sur le terrain permet de recueillir de telles informations.

7) J'ai pu constater par la même occasion que les cadres nationaux des services techniques traditionnels tiennent un discours différent selon qu'ils sont en présence ou non des évaluateurs (qu'ils considèrent eux même comme des bailleurs de fonds). C'est ainsi que lors de mon long séjour j'ai entendu dire par ces derniers que le PAGT équivalait à un "Prêt À Jeter", car ils ne tirent aucun intérêt de celui-ci comparativement au projet classique (pas de per diem, pas de bons d'essence...). Ceci, $\mathrm{du}$ reste, traduit le climat de tension qui règne entre ces cadres et les responsables $\mathrm{du}$ projet. Il existe de nombreux exemples illustrant les contradictions entre les différents acteurs. Ces faits ne sont jamais saisis par les évaluateurs.

81 8) L'attitude non seulement des animateurs et cadres du projet (recrutés avec un statut de contractuels sur le marché du travail), mais aussi celle de l'assistance technique étrangère a retenu mon attention. J'ai observé que ces deux parties se sont mobilisées pour orienter les villageois dans la restitution devant les évaluateurs d'un discours officiel pouvant favoriser la poursuite du projet. Cela se comprend car, de la survie de ce dernier, dépend le renouvellement de leur contrat d'embauche.

82 9) L'analyse du rapport final présenté par les membres de cette mission ex-post me ramène bien à mon propos de départ puisque celle ci s'est reposée spécialement sur les rapports et informations officielles du projet et qu'elle a eu à réaliser des visites de terrain très brèves en compagnie de l'encadrement (animateurs du projet).

Ce que je peux dire sur les résultats présentés par cette mission technico-économique (évaluation des réalisations) est très simple: celle-ci n'a malheureusement pas pu atteindre ses objectifs, car ses membres ont eux-mêmes souligné, avant leur départ de Niamey, qu'ils avaient trouvé une situation trop confuse qui ne facilitait pas leur tâche. Cela apparaît très vite à l'analyse du rapport final, d'où il transparaît que l'on n'a pas été au fond des choses. La restitution orale avait au moins eu le mérite de susciter un débat On peut se poser la question de savoir ce qui s'est réellement passé, notamment pourquoi il n'est nulle part fait allusion aux travaux de conservation du milieu, et maintien du potentiel et équipements villageois, qui étaient pour tant quelques unes des activités centrales du projet.

De ces considérations découlent peu de recommandations pratiques, nous sommes dans l'ordre du constat et de la compréhension des problèmes et non dans celui de l'action et de la transformation. "Il n'existe pas de recette miracle que l'anthropologue peut se permettre de donner à l'agent de développement ou à l'opérateur des projets "(J.P. 
Olivier de Sardan). J'estime simplement qu'une enquête qualitative de longue durée est nécessaire pour l'amélioration de la mise en œuvre des opérations de développement.

\section{NOTES}

1.Il ne s'agit pas ici d'un compte rendu de tout ce qui a été dit lors de cette rencontre mais seulement d'une analyse de contenu. Celle-ci ne pourra être complète que par le rappel de quelques conclusions ayant provoqué des débats entre les différents partenaires et susceptibles d'être l'objet d'une critique par l'anthropologue qui a séjourné sur le terrain. 